\title{
Nomograms predicting the overall survival and cancer-specific survival of patients with stage IIIC1 cervical cancer
}

Yifan Feng ${ }^{1 \dagger}$, Ye Wang ${ }^{2 \dagger}$, Yangqin Xie ${ }^{1}$, Shuwei Wu', Yuyang $\mathrm{Li}^{1}$ and Min $\mathrm{Li}^{1 *}$

\begin{abstract}
Background: To explore the factors that affect the prognosis of overall survival (OS) and cancer-specific survival (CSS) of patients with stage IIIC1 cervical cancer and establish nomogram models to predict this prognosis.

Methods: Data from patients in the Surveil-lance, Epidemiology, and End Results (SEER) programme meeting the inclusion criteria were classified into a training group, and validation data were obtained from the First Affiliated Hospital of Anhui Medical University from 2010 to 2019. The incidence, Kaplan-Meier curves, OS and CSS of patients with stage IIIC1 cervical cancer in the training group were evaluated. Nomograms were established according to the results of univariate and multivariate Cox regression models. Harrell's C-index, calibration plots, receiver operating characteristic (ROC) curves and decision-curve analysis (DCA) were calculated to validate the prediction models.

Results: The incidence of pelvic lymph node metastasis, a high-risk factor for the prognosis of cervical cancer, decreased slightly over time. Eight independent prognostic variables were identified for OS, including age, race, marriage status, histology, extension range, tumour size, radiotherapy and surgery, but only seven were identified for CSS, with marriage status excluded. Nomograms of OS and CSS were established based on the results. The Cindexes for the nomograms of OS and CSS were 0.687 and 0.692, respectively, using random sampling of SEER data sets and 0.701 and 0.735 , respectively, using random sampling of external data sets. The AUCs for the nomogram of OS were 0.708 and 0.705 for the SEER data sets and 0.750 and 0.750 for the external data sets, respectively. In addition, AUCs of 0.707 and 0.709 were obtained for the nomogram of CSS when validated using SEER data sets, and 0.788 and 0.785 when validated using external data sets. Calibration plots for the nomograms were almost identical to the actual observations. The DCA also indicated the value of the two models.
\end{abstract}

Conclusions: Eight independent prognostic variables were identified for OS. The same factors predicted CSS, with the exception of the marriage status. Both OS and CSS nomograms had good predictive and clinical application value after validation. Notably, tumour size had the largest contribution to the OS and CSS nomograms.

Keywords: Cervical cancer, Overall survival, Cancer-special survival, Nomogram, FIGO

\footnotetext{
* Correspondence: liminzhi@ahmu.edu.cn

${ }^{+}$Yifan Feng and Ye Wang contributed equally to this work.

'Department of Obstetrics and Gynecology, the First Affiliated Hospital of Anhui Medical University, Hefei 230022, China

Full list of author information is available at the end of the article
}

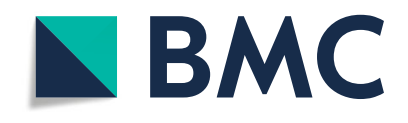

(C) The Author(s). 2021 Open Access This article is licensed under a Creative Commons Attribution 4.0 International License, which permits use, sharing, adaptation, distribution and reproduction in any medium or format, as long as you give appropriate credit to the original author(s) and the source, provide a link to the Creative Commons licence, and indicate if changes were made. The images or other third party material in this article are included in the article's Creative Commons licence, unless indicated otherwise in a credit line to the material. If material is not included in the article's Creative Commons licence and your intended use is not permitted by statutory regulation or exceeds the permitted use, you will need to obtain permission directly from the copyright holder. To view a copy of this licence, visit http://creativecommons.org/licenses/by/4.0/ The Creative Commons Public Domain Dedication waiver (http://creativecommons.org/publicdomain/zero/1.0/) applies to the data made available in this article, unless otherwise stated in a credit line to the data. 


\section{Background}

Cervical cancer is the most common malignant tumour of the female reproductive system, and the fourth most common malignant tumour in women, after breast cancer, colorectal cancer and lung cancer [1]. In 2018, approximately 570,000 women were diagnosed with cervical cancer and 311,000 women died from it [1]. Persistent carcinogenic human papillomavirus infection is the main cause of cervical cancer development [2]. Fortunately, due to the development of the HPV vaccine, a treatment protecting against cervical precancerous lesions, the incidence and mortality of cervical cancer in developed countries are gradually decreasing [3]. However, in developing countries, cervical cancer is still one of the most common cancers and the main cause of cancer-related death in women [4]. For example, in China, the incidence and mortality of cervical cancer are increasing significantly, especially among young women [5]. Due to the substantial economic burden of cervical cancer screening and vaccination programmes, many women are still suffering from HPV infection and its related cervical cancer [6].

The International Federation of Obstetrics and Gynecology (FIGO) stage is a systematic staging system based on a clinical examination [7]. In 2018, FIGO made important adjustments to the cervical cancer staging system [7]. Compared to the 2014 FIGO staging system, several changes were introduced. (a) Horizontal infiltration width no longer affects the stage. (b) An additional stage, IB3 was added to stage IB. In the revised system for stage IB disease, for every $2 \mathrm{~cm}$ increase in tumour size, the substage increases. Tumours smaller than $2 \mathrm{~cm}$ are classified as IB1, tumours greater than or equal to 2 $\mathrm{cm}$ and less than $4 \mathrm{~cm}$ are classified as IB2, tumours greater than or equal to $4 \mathrm{~cm}$ are classified as IB3. (c) Pelvic lymph node metastasis or paraaortic lymph node metastasis are directly classified as stage IIIC1/2 [7, 8].

The FIGO staging system is most frequently used to assess the prognosis of patients with cervical cancer. The new FIGO stage reflects the important effect of lymph node metastasis on the prognosis of cervical cancer patients [9]. However, the survival rate is heterogeneous for patients with the same stage. The prediction of prognosis using the FIGO staging system is not sufficiently comprehensive, and the accuracy must be improved [10,11].

A trend is to use nomograms to build cancer prediction models, because nomograms simplify a large number of complex factors into a single simple numerical estimation model to predict the probability of events [11]. Currently, few prognostic analyses of stage IIIC1 cervical cancer have been performed, and no nomogram has been established for patients with stage IIIC1 cervical cancer.
The purpose of this study was to explore the factors that affect the prognosis of patients with stage IIIC1 cervical cancer and establish nomogram models to predict the prognosis of stage IIIC1 cervical cancer.

\section{Materials and methods \\ Data source}

This retrospective observational study was conducted with data from the Surveil-lance, Epidemiology, and End Results (SEER) programme. The SEER database is a publicly available, federally funded cancer reporting system [12]. No cases extracted from the SEER database contain any personally identifying information.

One external validation set was generated to validate the nomogram in the present analysis, and the data were obtained from the First Affiliated Hospital of Anhui Medical University from 2010 to 2019; all patients in this dataset were pathologically diagnosed with cervical cancer. All patients under closed follow-up, every 3 months for the first 2 years, every 6 months for the next 3 years, and annually thereafter. This study was approved by Anhui Medical University Ethics Committee.

\section{Inclusion criteria}

Inclusion criteria were patients with a pathological cervical cancer diagnosis who were included in the SEER database from 2004 to 2015; cervical cancer was the first primary tumour; no stage IV disease; all patients underwent surgery and were evaluated positive for pelvic lymph node metastasis; age $\geq 18$ years; patients who died within 1 month; information about race, differentiation, surgery, marriage status, tumour size, extension range was complete; and the histopathological diagnosis was squamous cell carcinoma, adenocarcinoma or adenosquamous carcinoma utilizing ICD-O-3 codes, with poor / moderate / well differentiation (shown in Fig. 1). Patients with negative lymph nodes were selected in the same manner to compare the data with patients with pelvic lymph node metastasis. The external validation set was selected using the same criteria.

\section{Statistical analysis}

Categorical variables are described as counts and percentages. Kaplan-Meier curves and log-rank tests were used to draw overall survival (OS) curves and cancerspecific survival (CSS) curves in groups with different lymph nodes metastasis statuses. Additionally, univariate and multivariate Cox regression models were employed to estimate hazard ratios (HRs) and exact 95\% confidence intervals (CIs) to analyse the prognostic factors for stage IIIC1 cervical cancer. OS was the primary endpoint outcome from the date of diagnosis to the date of death or the latest follow-up. CSS was the special 


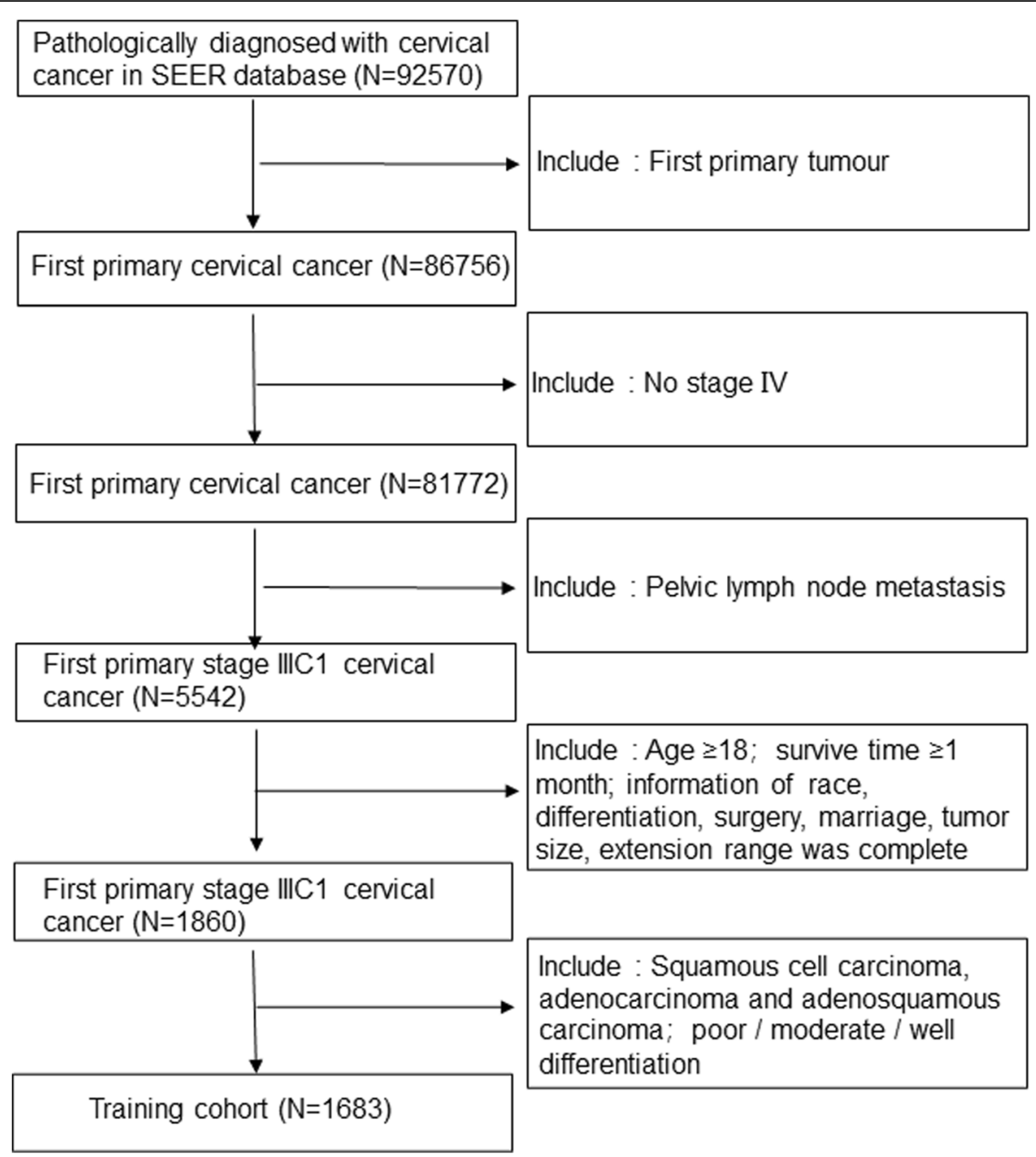

Fig. 1 Study selection process

endpoint outcome from the date of diagnosis to the date of death from cervical cancer or the latest follow-up.

Significant prognostic factors of OS and CSS in the Cox proportional hazards regression model were used to build the nomograms to predict the 3- and 5-year OS and CSS rates. Harrell's C-index and receiver operating characteristic (ROC) curves were calculated to measure the accuracy of the prediction models. Calibration plots show the relationship between the predicted probability and the actual outcome. Finally, decision-curve analysis (DCA) was applied to evaluate the clinical applicability of the constructed nomogram by quantifying the net improved benefits at various threshold probabilities. All statistical analyses and plots were performed using SPSS 23.0 (Chicago, IL, USA) and R version 3.6.2 (http://www. R-project ct.org/). $P$ values $<0.05$ were considered significant. All methods were performed in accordance with the relevant guidelines and regulations.

\section{Results}

Incidence and survival analyses

From 2004 to 2015, 1638 patients with cervical cancer exhibited pelvic lymph node metastasis (21.17\%). First, the incidence of lymph node metastasis in patients with cervical cancer decreased slightly from 2004 to 2015, as shown in Fig. 2a. According to the log-rank test of either OS or CSS, pelvic lymph node metastasis was a high-risk factor for the prognosis of patients with cervical cancer (both $p<0.001$ ), and Kaplan-Meier survival curves also confirmed the effect of lymph node metastasis on the prognosis, as shown in Fig. 2b-c.

\section{Patients characteristics}

The training cohort included, a total of 1683 patients diagnosed with cervical cancer between 2004 and 2015 . For the validation cohort, 148 patients met the criteria in the First Affiliated Hospital of Anhui Medical University between 2009 and 2019, 125 of whom were in active 


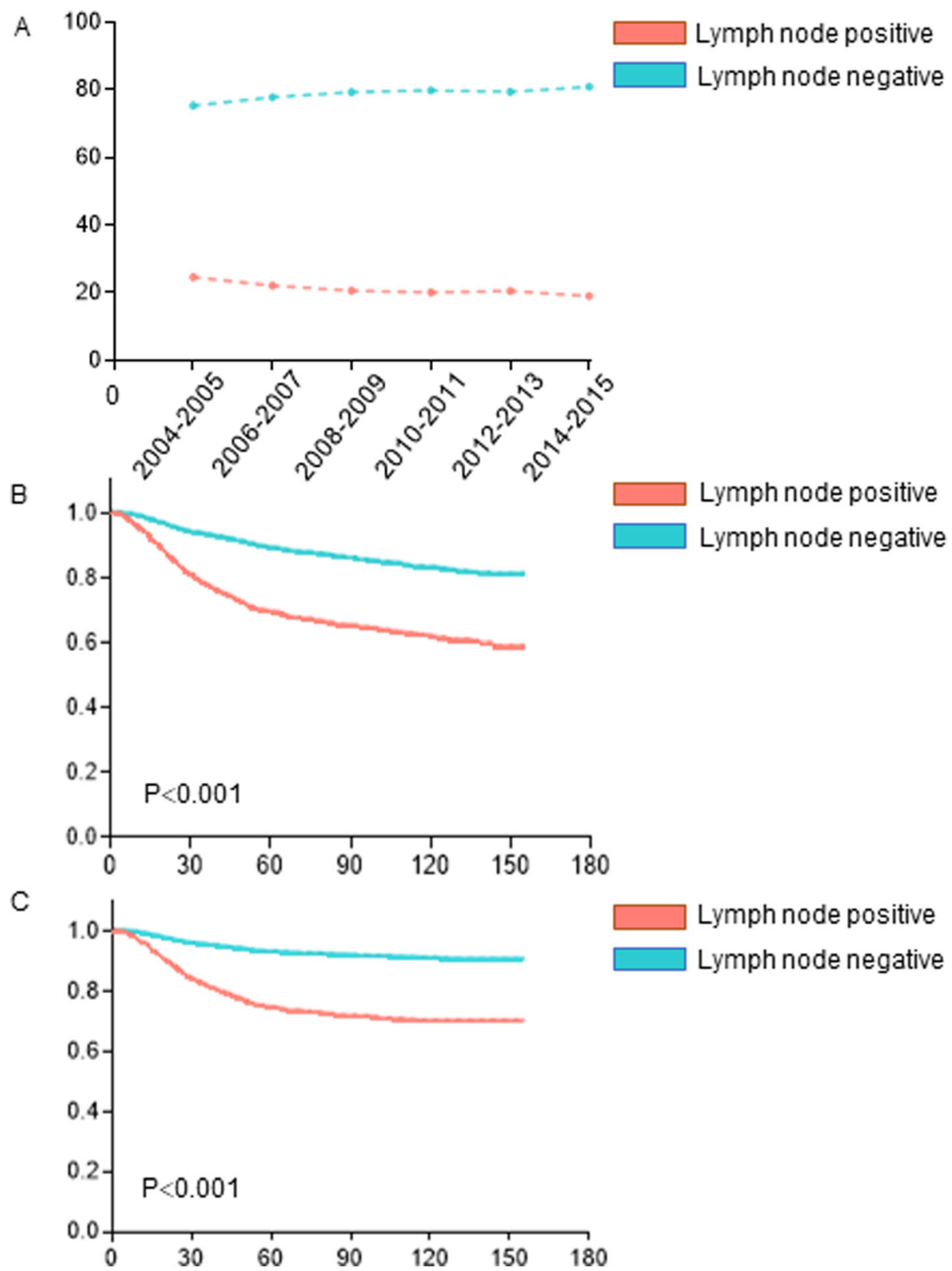

Fig. 2 Incidence and survival analyses of lymph node metastasis. a Incidence of lymph node metastasis; Kaplan-Meier survival curves of OS (b) and CSS (c) showing the association between lymph node metastasis and prognosis

follow-up, and $15.54 \%$ were lost to follow-up. The majority of patients in the training cohort were middleaged women $(57.80 \%)$, and patients in the validation cohort were middle-aged women, similar to the training cohort. Regarding tumour characteristics, squamous cell carcinoma, confined to the cervix uteri / uterus and with tumour size $\geq 4$ was the main diagnosis in both cohorts. However, the distributions of poor differentiated tumours distributions were 52.70 , and $22.40 \%$ respectively in training and validation cohorts, respectively. Details are provided in Table 1 . The OS rate of the training and validation cohorts were $30.30,32.80 \%$, respectively. The
CSS rates of the training and validation cohorts were $23.10,25.60 \%$, respectively.

\section{Prognostic factors for OS and CSS}

Univariate and multivariate Cox proportional hazard regression analyses were used to calculate the prognostic factors for OS and CSS. The results of the univariate Cox proportional hazard regression analysis are shown in Table 2, and the results of the multivariate Cox proportional hazard regression analysis are shown in forest plots (Fig. 3a-b). Age, race, marriage status, histology, extension range, tumour 
Table 1 Patient characteristics in the training cohort and validation cohort

\begin{tabular}{|c|c|c|c|c|}
\hline & \multicolumn{2}{|c|}{ Training cohort $(N=1683)$} & \multicolumn{2}{|c|}{ Validation cohort $(N=125)$} \\
\hline & $\mathrm{N}$ & Percent & $\mathrm{N}$ & Percent \\
\hline \multicolumn{5}{|l|}{ Age } \\
\hline$x<30$ & 132 & 7.80 & 3 & 2.40 \\
\hline $30 \leq x<50$ & 972 & 57.80 & 82 & 65.60 \\
\hline $50 \leq x<70$ & 494 & 29.40 & 40 & 32.00 \\
\hline$x \geq 70$ & 85 & 5.10 & 0 & 0.00 \\
\hline \multicolumn{5}{|l|}{ Race } \\
\hline White & 1334 & 79.30 & 0 & 0.00 \\
\hline Black & 134 & 8.0 & 0 & 0.00 \\
\hline Others & 215 & 12.80 & 125 & 100.00 \\
\hline \multicolumn{5}{|l|}{ Marital status } \\
\hline Married & 834 & 49.60 & 112 & 89.60 \\
\hline Single & 518 & 30.80 & 0 & 0.00 \\
\hline Divorced / Separated & 238 & 14.10 & 7 & 5.60 \\
\hline Widowed & 93 & 5.50 & 6 & 4.80 \\
\hline \multicolumn{5}{|l|}{ Histology } \\
\hline Squamous cell carcinoma & 1211 & 72.00 & 99 & 79.20 \\
\hline Adenocarcinoma & 337 & 20.00 & 25 & 20.00 \\
\hline Adenosquamous & 135 & 8.00 & 1 & 0.80 \\
\hline \multicolumn{5}{|l|}{ Differentiation } \\
\hline Well differentiation & 79 & 4.70 & 4 & 3.20 \\
\hline Moderate differentiation & 717 & 42.60 & 93 & 74.40 \\
\hline Poor differentiation & 887 & 52.70 & 28 & 22.40 \\
\hline \multicolumn{5}{|l|}{ Extension range } \\
\hline Confined to the cervix uteri / uterus & 1036 & 61.60 & 91 & 72.80 \\
\hline Extension beyond uterus & 647 & 38.40 & 34 & 27.20 \\
\hline \multicolumn{5}{|l|}{ Tumour size (cm) } \\
\hline$x<2$ & 214 & 12.70 & 6 & 4.80 \\
\hline $2 \leq x<4$ & 623 & 37.00 & 47 & 37.60 \\
\hline$x \geq 4$ & 846 & 50.30 & 72 & 57.60 \\
\hline \multicolumn{5}{|l|}{ Radiotherapy } \\
\hline Yes & 1433 & 85.10 & 92 & 73.60 \\
\hline No & 250 & 14.90 & 33 & 26.40 \\
\hline \multicolumn{5}{|l|}{ Chemotherapy } \\
\hline Yes & 1337 & 79.40 & 116 & 92.80 \\
\hline No & 346 & 20.60 & 9 & 7.20 \\
\hline \multicolumn{5}{|l|}{ Surgery } \\
\hline Preserve uterus & 341 & 20.30 & 0 & 0.00 \\
\hline Hysterectomy & 1342 & 79.70 & 125 & 100.00 \\
\hline \multicolumn{5}{|l|}{ Survival state } \\
\hline Survive & 1173 & 69.70 & 84 & 67.20 \\
\hline Dead of other cause & 121 & 7.20 & 9 & 7.20 \\
\hline Dead of cervical cancer & 389 & 23.10 & 32 & 25.60 \\
\hline
\end{tabular}


Table 2 Univariate analysis of OS and CSS in the training cohort

\begin{tabular}{|c|c|c|c|c|}
\hline \multirow[t]{2}{*}{ Characteristics } & \multicolumn{2}{|l|}{ OS } & \multicolumn{2}{|l|}{ CSS } \\
\hline & Hazard ratios $(95 \% \mathrm{Cl})$ & $\mathbf{P}$ & Hazard ratios $(95 \% \mathrm{Cl})$ & $\mathbf{P}$ \\
\hline \multicolumn{5}{|l|}{ Age } \\
\hline$x<30$ & Reference & & Reference & \\
\hline $30 \leq x<50$ & $0.628(0.461-0.855)$ & 0.003 & $0.597(0.427-0.835)$ & 0.003 \\
\hline $50 \leq X<70$ & $0.859(0.622-1.187)$ & 0.358 & $0.722(0.506-1.031)$ & 0.073 \\
\hline$x \geq 70$ & $1.737(1.167-2.587)$ & 0.007 & $1.025(0.623-1.686)$ & 0.922 \\
\hline \multicolumn{5}{|l|}{ Race } \\
\hline White & Reference & & Reference & \\
\hline Black & $1.363(1.025-1.810)$ & 0.033 & $1.512(1.106-2.067)$ & 0.010 \\
\hline Others & 0.835 (0.628-1.109) & 0.212 & $0.773(0.552-1.083)$ & 0.134 \\
\hline \multicolumn{5}{|l|}{ Marriage } \\
\hline Married & Reference & & Reference & \\
\hline Single & 1.089 (0.887-1.337) & 0.415 & 1.030 (0.818-1.297) & 0.802 \\
\hline Divorced / Separated & $1.369(1.071-1.751)$ & 0.012 & 1.193 (0.897-1.588) & 0.225 \\
\hline Widowed & $1.820(1.301-2.546)$ & $<0.001$ & $1.161(0.740-1.823)$ & 0.516 \\
\hline \multicolumn{5}{|l|}{ Histology } \\
\hline Squamous cell carcinoma & Reference & & Reference & \\
\hline Adenocarcinoma & $1.285(1.043-1.581)$ & 0.018 & $1.345(1.063-1.701)$ & 0.014 \\
\hline Adenosquamous & $1.284(0.944-1.746)$ & 0.112 & $1.298(0.912-1.848)$ & 0.147 \\
\hline \multicolumn{5}{|l|}{ Differentiation } \\
\hline Well differentiation & Reference & & Reference & \\
\hline Moderate differentiation & $1.133(0.707-1.815)$ & 0.603 & $1.326(0.753-2.335)$ & 0.328 \\
\hline Poor differentiation & $1.406(0.884-2.236)$ & 0.150 & $1.524(0.871-2.667)$ & 0.140 \\
\hline \multicolumn{5}{|l|}{ Extension range } \\
\hline Confined to the cervix uteri / uterus & Reference & & Reference & \\
\hline Extension beyond uterus & $2.044(1.718-2.432)$ & $<0.001$ & $2.013(1.650-2.456)$ & $<0.001$ \\
\hline \multicolumn{5}{|l|}{ Tumour size $(\mathrm{cm})$} \\
\hline$x<2$ & Reference & & Reference & \\
\hline $2 \leq X<4$ & $2.323(1.498-3.602)$ & $<0.001$ & $2.933(1.650-5.214)$ & $<0.001$ \\
\hline$x \geq 4$ & $4.574(2.998-6.980)$ & $<0.001$ & $6.407(3.672-11.180)$ & $<0.001$ \\
\hline \multicolumn{5}{|l|}{ Radiotherapy } \\
\hline Yes & Reference & & Reference & \\
\hline No & $1.314(1.047-1.651)$ & 0.019 & $1.232(0.944-1.608)$ & 0.125 \\
\hline \multicolumn{5}{|l|}{ Chemotherapy } \\
\hline Yes & Reference & & Reference & \\
\hline No & $0.906(0.729-1.126)$ & 0.374 & $0.778(0.599-1.011)$ & 0.060 \\
\hline \multicolumn{5}{|l|}{ Surgery } \\
\hline Preserve uterus & Reference & & Reference & \\
\hline Hysterectomy & $0.537(0.443-0.652)$ & $<0.001$ & $0.504(0.405-0.627)$ & $<0.001$ \\
\hline
\end{tabular}

size, radiotherapy and surgery were all independent prognostic factors for OS. Independent prognostic factors for CSS were the same as those for OS, except the marriage status.

\section{Nomograms}

Based on the prognostic factors for OS and CSS derived from the Cox proportional hazard regression analyses, OS and CSS nomograms were established and are shown 


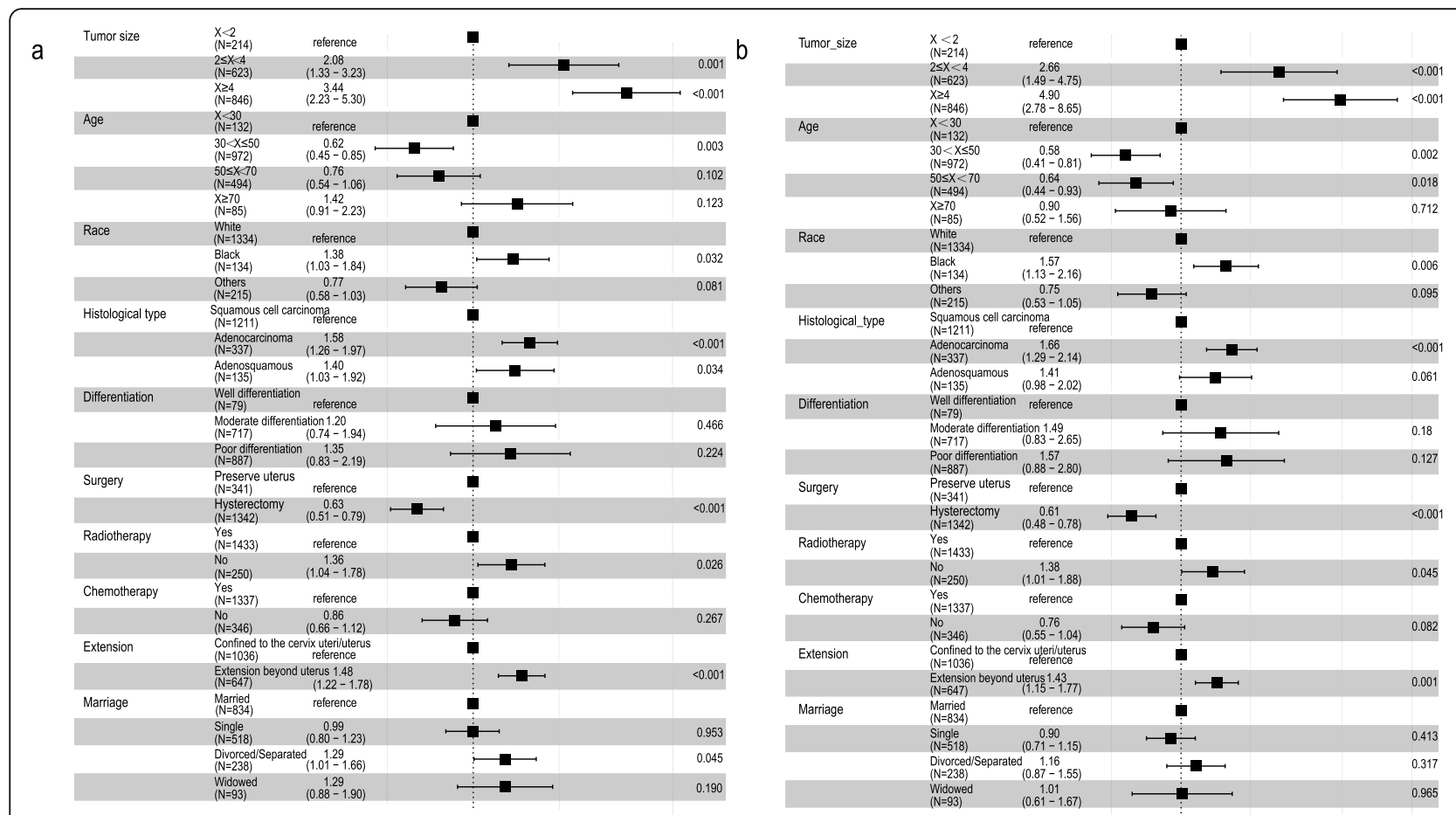

Fig. 3 Forest plots of the multivariate analysis of OS (a) and CSS (b) in patients with stage IIIC1 cervical cancer

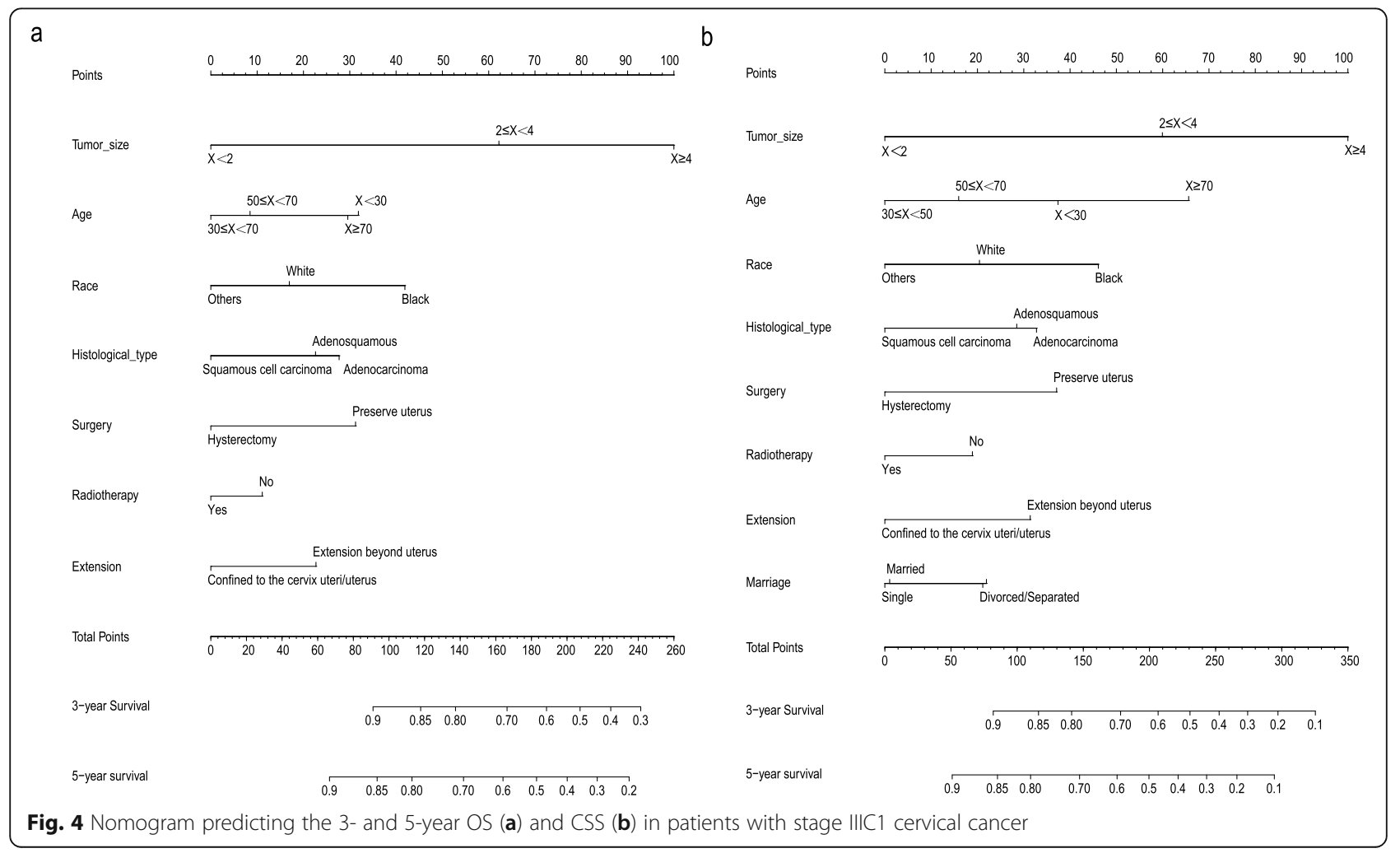


in Fig. 4. The C-indexes for the nomograms of OS and CSS were 0.687 and 0.692 , respectively, using random sampling of SEER data sets and 0.701 and 0.735 , respectively, when random sampling of external data sets. Calibration plots for the nomograms showed that the predicted 3- and 5-year OS and CSS probabilities for the training and validation sets were almost identical to the actual observations, as displayed in Fig. 5. As shown in the ROC curves for the nomogram prediction models presented in Fig. 6, the 3- and 5-year AUCs for the nomogram of OS were 0.708 and 0.705 , respectively, for SEER data sets and 0.750 and 0.750 , respectively, for external data sets. In addition, AUCs were 0.707 and 0.709 for the nomogram of CSS when validated using SEER data sets, and 0.788 and 0.785 when validated using external data sets. All the AUCs indicated a good discrimination ability of the model. The DCA also showed the value of the two models. The net benefit of our prognostic models was larger than that in the other two scenarios (all screening or nonscreening) in a wide range of threshold probabilities as displayed in Fig. 7.

\section{Discussion}

Researchers have discovered that pelvic lymph node metastasis is a high-risk factor for patients with cervical cancer, which has been confirmed in many studies [13, 14]. Until 2018, FIGO agreed that lymph node metastasis had the greatest effect on the prognosis of patients diagnosed with cervical cancer, except for spreading to adjacent pelvic organs or distant organs. However, a study by Xiaoliang Liu found that the survival rate is heterogeneous in patients with stage IIIC1 cervical cancer, and tumour size, extension range, and other factors exert significant effects on the prognosis of stage IIIC1 cervical cancer [15]. Therefore, we included 10 variables from the SEER database to analyse the factors that affect the prognosis of patients with stage IIIC1 cervical cancer. Furthermore, previous studies documented that these 10 variables were significantly associated with the prognosis of cervical cancer. For this reason, univariate and multivariate Cox proportional hazard regression analyses were performed for all these 10 variables [1517].

Then, we established OS and CSS nomograms based on the results of the multivariate Cox proportional hazard regression analysis. The factors in the OS nomogram included age, race, marriage status, tumour size, histology, extension range, surgery, and radiotherapy. For the CSS nomogram, only marriage status was excluded. Previous studies of the effect of marital status on cancer have shown that married patients have advantages in terms of the early diagnosis of cancer, which included cervical cancer [18]. In addition, married patients are able to receive more comprehensive adjuvant treatment, leading to a better prognosis of cervical cancer [19]. However, we found that marriage status was not an independent prognostic factor for CSS in patients with stage IIIC1 cervical cancer. Notably, unmarried patients
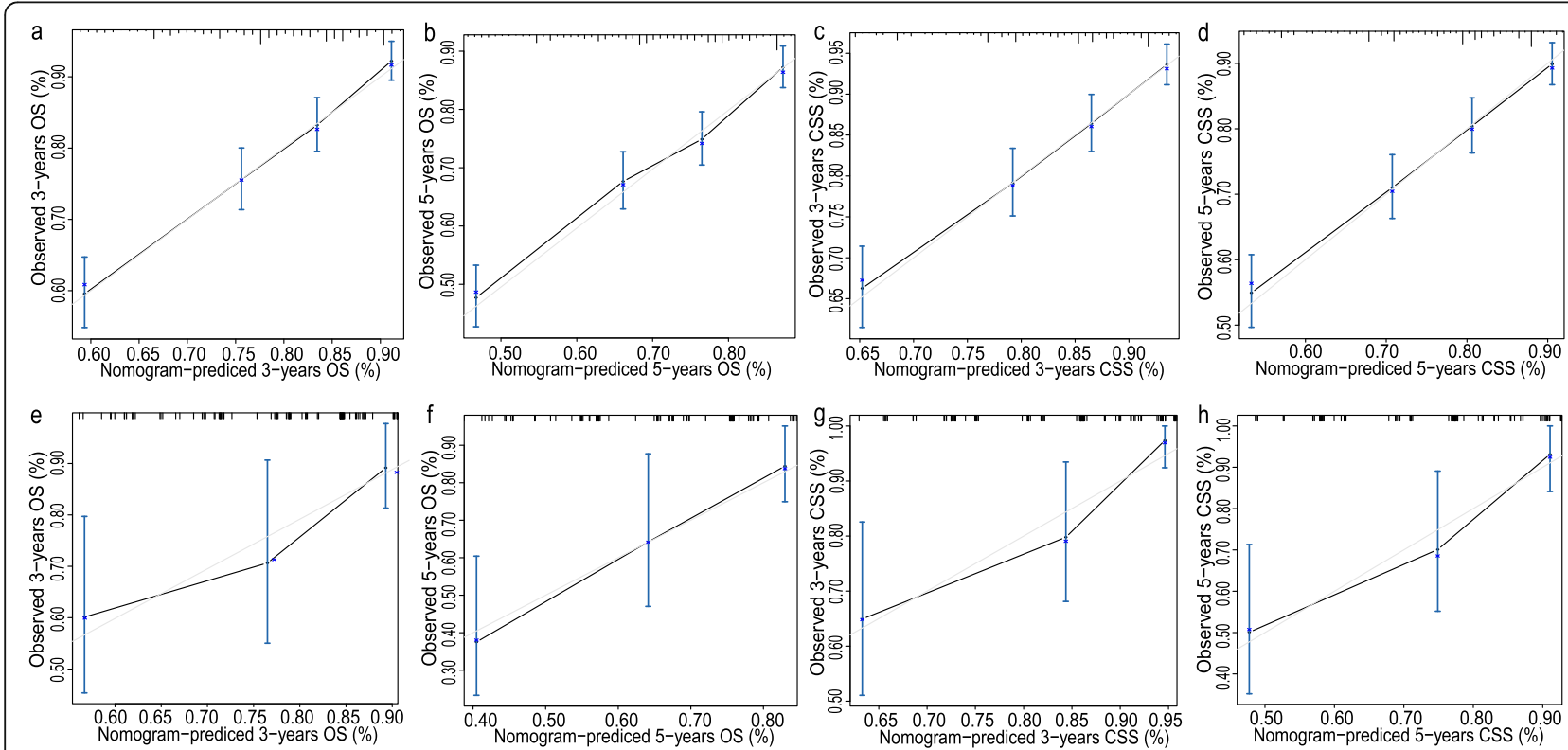

Fig. 5 Calibration plots for (a) the 3-year OS nomogram in the training cohort; $\mathbf{b}$ the 5-year OS nomogram in the training cohort; $\mathbf{c}$ the 3-year CSS nomogram in the training cohort; $\mathbf{d}$ the 5-year CSS nomogram in the training cohort; $\mathbf{e}$ the 3-year OS nomogram in the validation cohort; $\mathbf{f}$ the 5-year OS nomogram in the validation cohort; $\mathbf{g}$ the 3-year CSS nomogram in the validation cohort; $\mathbf{h}$ the 5-year CSS nomogram in the validation cohort 

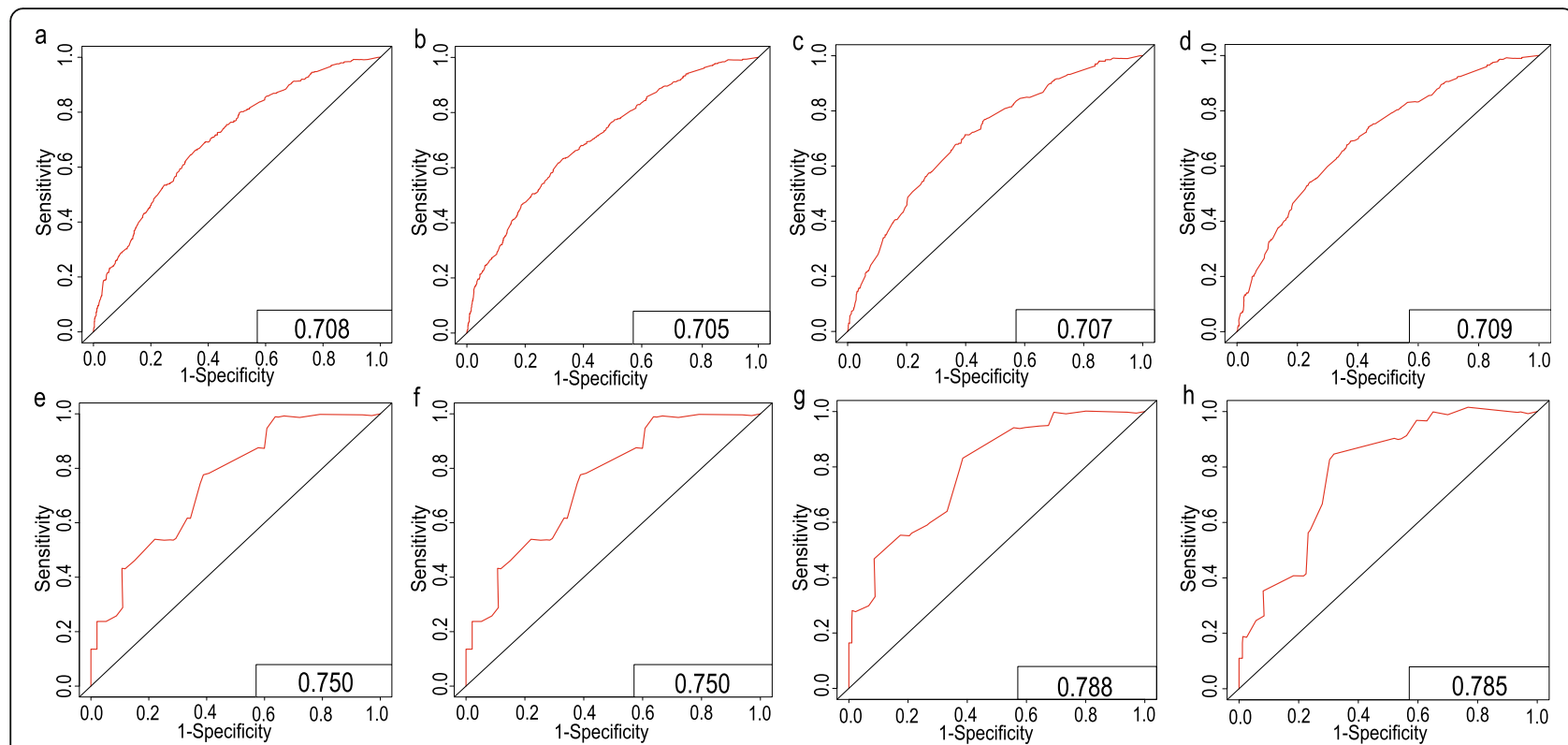

Fig. 6 ROC curves for (a) the 3-year OS nomogram in the training cohort; $\mathbf{b}$ the 5-year OS nomogram in the training cohort; c the 3-year CSS nomogram in the training cohort; $\mathbf{d}$ the 5-year CSS nomogram in the training cohort; e the 3-year OS nomogram in the validation cohort; $\mathbf{f}$ the 5-year OS nomogram in the validation cohort; $\mathbf{g}$ the 3-year CSS nomogram in the validation cohort; $\mathbf{h}$ the 5-year CSS nomogram in the validation cohort

also had diabetes, hypertension, cardiovascular disease and other comorbidities [20]. These additional diseases increase the risk of patients died from other causes and may be the main reason why marriage status was not an independent prognostic factor for CSS in patients with stage IIIC1 cervical cancer.

Our nomograms are highly innovative and practical. First, although nomograms for cervical cancer have been widely used [17, 21], a nomogram for stage IIIC1 cervical cancer is not available. Second, in contrast to the FIGO stage, patient demographics (age, race and marriage status), tumour characteristics (tumour size, histology, and extension range) and treatment (surgery and radiotherapy) which were independent prognostic factors for OS or CSS were included in our nomograms. And these variables are easily obtained in the clinic. Therefore, our nomograms could reduce the bias caused by patient demographics and different treatments when predicting the prognosis of stage IIIC1 cervical cancer compare to the FIGO stage. Third, our nomograms were verified using external data sets. This process can test the predictive ability of the nomogram in different groups of people and judge its applicability to various groups of people [22].

The C-indexes of the nomograms of random sampling of SEER and external data sets were all range from 0.65 to 0.75 , which were acceptable and indicating that our nomograms have favourable discrimination ability [23, 24]. In addition, our calibration plots fit well with the 45-degree line, which means our nomograms have a fine calibration [25]. Therefore, our nomograms have good calibration in predicting 3- and 5-year OS and CSS. And DCA was performed to evaluate the clinical applicability of the constructed nomograms when quantifying the net improvement benefits under different threshold probabilities [26]. After validation, DCA confirmed that our nomograms have better clinical benefits and utility in predicting the survival of patients with stage IIIC1 cervical cancer.

Notably, tumour size had the largest contribution to the OS and CSS nomograms.. In cervical cancer, the effect of tumor size on prognosis in stage IB and stage II has been confirmed and shown in the FIGO staging system [27, 28]. According to the multivariate Cox proportional hazard regression analysis, as the tumour size increases, the prognosis of patients with stage IIIC1 disease becomes significantly worse. Meanwhile, imaging data can provide evidence of the FIGO stage [7]. Studies on the application of imaging to assess the tumour size of cervical cancer before surgery show that the diagnostic power of imaging is obviously stronger than a clinical assessment [29], especially MRI, depending on its superior contrast resolution, which can visualize the tumour volume and size [30]. We conclude that compared to other pathological characteristics, the effect of tumour size on the prognosis of cervical cancer is consistent across most stages. Further research revealed the value of tumour size as a prognostic indicator of stage IIIC1 cervical cancer. Therefore, we suggest that stage IIIC1 cervical cancer should be further divided into three substages and treated with different strategies according to tumour size. 


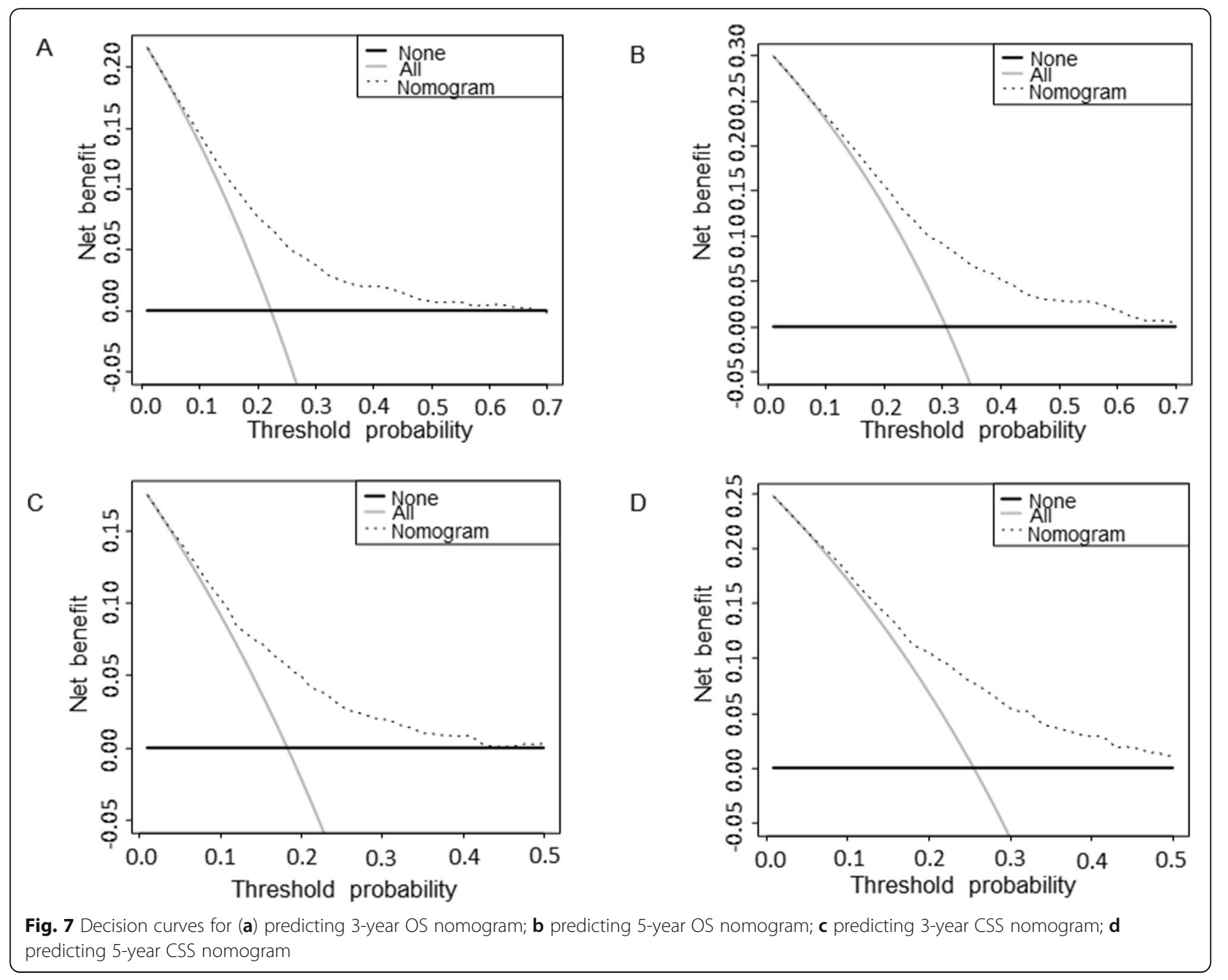

Although the nomograms were verified using an external data set, our study still has some limitations. First, as a retrospective study, this research filtered data from data sets and excluded patients with missing data for the collected variables, leading to selection bias. Second, some key indicators are lacking, especially the dosage of radiotherapy and details of chemotherapy regimens. For example, only "Yes" and "No" were shown in the SEER database for chemotherapy, leading to a weaken effect of chemotherapy on survival. Third, the insufficient sample size of the external data set and some missing data caused inadequate verification.

\section{Conclusions}

In conclusion, age, race, marriage status, histology, extension range, tumour size, radiotherapy and surgery were all independent prognostic factors for OS. The same factors predicted CSS, with the exception of the marriage status. In addition, OS and CSS nomograms were established in our study based on the results of a multivariate Cox proportional hazard regression analysis, and both had good predictive and clinical application value after validation. Notably, tumour size had the largest contribution to the OS and CSS nomograms.

\section{Abbreviations}

FIGO: International Federation of Obstetrics and Gynecology; SEER: The Surveil-lance, Epidemiology, and End Results; OS: Overall Survival; CSS: Cancer Special Survival; HR: Hazard Ratio; Cls: Confidence Intervals; ROC: Receiver Operating Characteristic; DCA: Decision-Curve Analysis

\section{Acknowledgements}

We thank all staff and participants of the Surveillance, Epidemiology, and End Results (SEER) program.

Authors' contributions

YFF, YW, ML designed the study. YFF, YW conducted the data analysis and YFF wrote the manuscript. SWW, YQX, and YYL collected the data. All authors were involved in interpreting the data and revising the manuscript. All authors agreed with the final publication. The author(s) read and approved the final manuscript.

Funding

There is no fund in this study. 


\section{Availability of data and materials}

One of the data of this study are available from the Surveillance, Epidemiology, and End Results (SEER) database (https://seer.cancer.gov/). But the availability of these data was restrictive, which were used under license for the current study (ID: 10086-Nov2019), and so are not publicly available. Based on reasonable request and with permission of SEER database, data are available from the corresponding authors. The external data were obtained from the First Affiliated Hospital of Anhui Medical University.

\section{Declarations}

\section{Ethics approval and consent to participate}

All cases extracted from the SEER database do not contain any personally identifying information. The external validation set are available to every researcher since all the patients were authorized by Anhui Medical University Ethics Committee when they signed to obey the data use agreement. This study was approved by Anhui Medical University Ethics Committee. The need of informed consent was waived by Anhui Medical University Ethics Committee for this retrospective study.

\section{Consent for publication}

NOT APPLICABLE.

\section{Competing interests}

The authors declared that no competing interest exists.

\section{Author details}

${ }^{1}$ Department of Obstetrics and Gynecology, the First Affiliated Hospital of Anhui Medical University, Hefei 230022, China. ${ }^{2}$ Department of General Surgery, the First Affiliated Hospital of Anhui Medical University, Hefei 230022, China

Received: 19 December 2020 Accepted: 16 April 2021 Published online: 23 April 2021

\section{References}

1. Arbyn M, Weiderpass E, Bruni L, de Sanjosé S, Saraiya M, Ferlay J, et al. Estimates of incidence and mortality of cervical cancer in 2018: a worldwide analysis. Lancet Glob Health. 2020;8(2):e191-203. https://doi.org/10.1016/ S2214-109X(19)30482-6.

2. Arbyn M, Bryant A, Beutels $P$, Martin-Hirsch PP, Paraskevaidis E, Van Hoof E, et al. Prophylactic vaccination against human papillomaviruses to prevent cervical cancer and its precursors. Cochrane Database Syst Rev. 2018;5: CD009069.

3. Siegel RL, KD Miller and A Jemal. Cancer statistics, 2018. CA Cancer J Clin, 2018 68(1): p. 7-30, DOl: https://doi.org/10.3322/caac.21442.

4. Bray F, Jemal A, Torre LA, Forman D, Vineis P. Long-term Realism and Costeffectiveness: Primary Prevention in Combatting Cancer and Associated Inequalities Worldwide. J Natl Cancer Inst. 2015;107(12):djv273.

5. Hu Z. And D Ma. The precision prevention and therapy of HPV-related cervical cancer: new concepts and clinical implications. Cancer Med. 2018; 7(10):5217-36. https://doi.org/10.1002/cam4.1501.

6. Plummer M, de Martel C, Vignat J, Ferlay J, Bray F, Franceschi S. Global burden of cancers attributable to infections in 2012 a synthetic analysis. Lancet Glob Health. 2016;4(9):e609-16. https://doi.org/10.1016/S2214-109X(1 6)30143-7.

7. Bhatla N, Aoki D, Sharma DN, Sankaranarayanan R. Cancer of the cervix uteri. Int J Gynaecol Obstet. 2018;143(Suppl 2):22-36. https://doi.org/10.1 002/ijgo.12611.

8. FIGO Committee on Gynecologic Oncology. FIGO staging for carcinoma of the vulva, cervix, and corpus uteri. Int J Gynaecol Obstet. 2014;125(2):97-8. https://doi.org/10.1016/j.ijgo.2014.02.003.

9. Waggoner SE. Cervical cancer. Lancet. 2003;361(9376):2217-25. https://doi. org/10.1016/S0140-6736(03)13778-6.

10. Wang C, Yang C, Wang W, Xia B, Li K, Sun F, et al. A prognostic Nomogram for cervical Cancer after surgery from SEER database. J Cancer. 2018;9(21): 3923-8. https://doi.org/10.7150/jca.26220.

11. Wan G, Gao F, Chen J, Li Y, Geng M, Sun L, et al. Nomogram prediction of individual prognosis of patients with hepatocellular carcinoma. BMC Cancer 2017;17(1):91. https://doi.org/10.1186/s12885-017-3062-6.
12. Doll KM, Rademaker A, Sosa JA. Practical guide to surgical data sets: surveillance, epidemiology, and end results (SEER) database. JAMA Surg. 2018;153(6):588-9. https://doi.org/10.1001/jamasurg.2018.0501.

13. Sevin BU, Lu Y, Bloch DA, Nadji M, Koechli OR, Averette HE. Surgically defined prognostic parameters in patients with early cervical carcinoma. A multivariate survival tree analysis. Cancer. 1996;78(7):1438-46. https://doi. org/10.1002/(SICI)1097-0142(19961001)78:7<1438::AID-CNCR10>3.0.CO;2-0.

14. Gien LT, Covens A. Lymph node assessment in cervical cancer: prognostic and therapeutic implications. J Surg Oncol. 2009;99(4):242-7. https://doi. org/10.1002/jso.21199.

15. Liu X, Wang W, Hu K, Zhang F, Hou X, Yan J, et al. A risk stratification for patients with cervical Cancer in stage IIIC1 of the 2018 FIGO staging system. Sci Rep. 2020;10(1):362. https://doi.org/10.1038/s41598-019-57202-3.

16. Matsuo K, Machida H, Mandelbaum RS, Konishi I, Mikami M. Validation of the 2018 FIGO cervical cancer staging system. Gynecol Oncol. 2019;152(1): 87-93. https://doi.org/10.1016/j.ygyno.2018.10.026.

17. Xie G, Wang R, Shang L, Qi C, Yang L, Huang L, et al. Calculating the overall survival probability in patients with cervical cancer: a nomogram and decision curve analysis-based study. BMC Cancer. 2020;20(1):833. https://doi. org/10.1186/s12885-020-07349-4.

18. Buja A, Lago L, Lago S, Vinelli A, Zanardo C, Baldo V. Marital status and stage of cancer at diagnosis: A systematic review. Eur J Cancer Care (Engl). 2018;27(1).

19. Huynh-Le MP, Klapheke A, Cress R, Mell LK, Yashar CM, Einck JP, et al. Impact of marital status on receipt of brachytherapy and survival outcomes in locally advanced cervical cancer. Brachytherapy. 2019;18(5):612-9. https:// doi.org/10.1016/j.brachy.2019.04.273.

20. Ramezankhani A, Azizi F, Hadaegh F. Associations of marital status with diabetes, hypertension, cardiovascular disease and all-cause mortality: a long term follow-up study. PLoS One. 2019;14(4):e0215593. https://doi.org/1 0.1371/journal.pone.0215593.

21. Marchetti C, De Felice F, Di Pinto A, Romito A, Musella A, Palaia I, et al. Survival Nomograms after curative Neoadjuvant chemotherapy and radical surgery for stage IB2-IIIB cervical Cancer. Cancer Res Treat. 2018;50(3):76876. https://doi.org/10.4143/crt.2017.141.

22. Iasonos A, Schrag D, Raj GV, Panageas KS. How to build and interpret a nomogram for cancer prognosis. J Clin Oncol. 2008;26(8):1364-70. https:// doi.org/10.1200/JCO.2007.12.9791.

23. Polterauer S, Grimm C, Hofstetter G, Concin N, Natter C, Sturdza A, et al. Nomogram prediction for overall survival of patients diagnosed with cervical cancer. Br J Cancer. 2012;107(6):918-24. https://doi.org/10.1038/bjc.2012.340.

24. Jiang S, Zhao R, Li Y, Han X, Liu Z, Ge W, et al. Prognosis and nomogram for predicting postoperative survival of duodenal adenocarcinoma: a retrospective study in China and the SEER database. Sci Rep. 2018;8(1):7940. https://doi.org/10.1038/s41598-018-26145-6.

25. Van Calster B, Nieboer D, Vergouwe Y, De Cock B, Pencina MJ, Steyerberg EW. A calibration hierarchy for risk models was defined: from utopia to empirical data. J Clin Epidemiol. 2016;74:167-76. https://doi.org/10.1016/j. jclinepi.2015.12.005.

26. Fitzgerald M, Saville BR, Lewis RJ. Decision curve analysis. JAMA. 2015;313(4): 409-10. https://doi.org/10.1001/jama.2015.37.

27. Landoni F, Maneo A, Colombo A, Placa F, Milani R, Perego P, et al. Randomised study of radical surgery versus radiotherapy for stage Ib-lla cervical cancer. Lancet. 1997;350(9077):535-40. https://doi.org/10.1016/S014 0-6736(97)02250-2.

28. Wagner AE, Pappas L, Ghia AJ, Gaffney DK. Impact of tumor size on survival in cancer of the cervix and validation of stage IIA1 and IIA2 subdivisions. Gynecol Oncol. 2013;129(3):517-21. https://doi.org/10.1016/j.ygyno.2013.03.008.

29. Mitchell DG, Snyder B, Coakley F, Reinhold C, Thomas G, Amendola M, et al. Early invasive cervical cancer: tumor delineation by magnetic resonance imaging, computed tomography, and clinical examination, verified by pathologic results, in the ACRIN 6651/GOG 183 intergroup study. J Clin Oncol. 2006;24(36):5687-94. https://doi.org/10.1200/JCO.2006.07.4799.

30. Nag S, Cardenes H, Chang S, Das IJ, Erickson B, Ibbott GS, et al. Varia M; image-guided brachytherapy working group. Proposed guidelines for image-based intracavitary brachytherapy for cervical carcinoma: report from image-guided brachytherapy working group. Int J Radiat Oncol Biol Phys. 2004;60(4):1160-72. https://doi.org/10.1016/j.jirobp.2004.04.032.

\section{Publisher's Note}

Springer Nature remains neutral with regard to jurisdictional claims in published maps and institutional affiliations. 